\title{
Correlates of spiritual wellbeing in persons living with Parkinson disease
}

\author{
Lindsay Penny Prizer ${ }^{1}$, Benzi M. Kluger ${ }^{2}$, Stefan Sillau², Maya Katz ${ }^{3}$, Nicholas Galifianakis ${ }^{3}$, \\ Janis M. Miyasaki ${ }^{4}$
}

${ }^{1}$ Division of General Medicine and Geriatrics, Emory University School of Medicine, Atlanta, GA, USA; ${ }^{2}$ Department of Neurology, University of Colorado School of Medicine, Aurora, CO, USA; ${ }^{3}$ Department of Neurology, University of California, San Francisco, CA, USA; ${ }^{4}$ Parkinson and Movement Disorders Program, Department of Medicine, University of Alberta, Edmonton, Canada

Contributions: (I) Conception and design: BM Kluger, JM Miyasaki, M Katz, N Galifianakis; (II) Administrative support: BM Kluger, JM Miyasaki, M Katz, N Galifianakis; (III) Provision of study materials or patients: BM Kluger, JM Miyasaki, M Katz, N Galifianakis; (IV) Collection and assembly of data: BM Kluger, JM Miyasaki, M Katz, N Galifianakis, S Sillau; (V) Data analysis and interpretation: S Sillau, BM Kluger, JM Miyasaki, LP Prizer; (VI) Manuscript writing: All authors; (VII) Final approval of manuscript: All authors.

Correspondence to: Lindsay Penny Prizer. Division of General Medicine and Geriatrics, Emory University School of Medicine, 1841 Clifton Road, $5^{\text {th }}$ Floor, Atlanta, GA 30329, USA. Email: lprizer@emory.edu.

Background: Spirituality influences chronic disease coping skills through both positive and negative means. Although previous research notes similar associations between faith and Parkinson disease (PD) management, little is known about spirituality in the context of PD management in a palliative setting. The purpose of this paper is to gain a better understanding of the association between spirituality and PD management to best maximize the effect of spiritual counseling in treatment of the disease.

Methods: This is a cross-sectional analysis of the baseline data of a larger randomized, controlled trial. Individuals with PD and their caregivers were recruited from three study sites with currently-operating outpatient movement disorders and palliative care programs. Correlations were conducted between FACIT total scores and subscales with hypothesized associative factors, such as quality of life (QOL), mood and cognition, advance care planning, and physical symptoms.

Results: Two-hundred and ten PD patients and 175 caregivers participated in the study. FACIT scores correlated positively with older age, existence of a caregiver, and involvement in support groups. Higher spirituality was associated with less impairment in QOL, lower anxiety, lower depression, fewer non-motor symptoms, reduced palliative symptoms, and less prolonged grief. There was no significant association between patient FACIT scores and the MDS UPDRS or MoCA scores.

Conclusions: These results highlight the influences of spirituality in PD management and further support the holistic, interdisciplinary care provided to PD patients through palliative care teams offering chaplaincy support to patients.

Keywords: Palliative care; supportive care; spiritual distress; Parkinson disease (PD); parkinsonism; spirituality

Submitted Aug 23, 2019. Accepted for publication Sep 19, 2019.

doi: 10.21037/apm.2019.09.13

View this article at: http://dx.doi.org/10.21037/apm.2019.09.13

\section{Introduction}

Spirituality often is a significant part of an individual's life. Yet, spirituality and its role in coping with chronic medical conditions has only recently been examined (1). Investigators have documented improved self-management behaviors, or to mental states such as happiness, optimism, and hope, all of which are related to better health outcomes associated with higher levels of spirituality (2). Research suggests that the positive association between spirituality and coping may be due to individuals with high levels of 
spirituality using more diverse and varied coping techniques to manage illness (3). Conversely, negative spiritual coping techniques (e.g., feeling punished by God) are associated with increased distress, depression, and impairment in quality of life (QOL) possibly resulting in hopelessness and despair (4). Despite this clear relationship between spirituality and coping with illness, little is known about spirituality and Parkinson disease (PD).

Exploratory research in PD and spirituality indicates that many PD patients rely on faith to find a deeper meaning and purpose in their illness. A cross-sectional study with PD patients in a Colorado clinic found that $59 \%$ reported using prayer to cope with their illness, and $67 \%$ of that patient subset felt prayer led to improvements in disease maintenance (5). Spiritual coping mechanisms, such as surrendering to a higher power, are frequently beneficial in managing symptoms and disease (6). A qualitative study on PD and spirituality noted distinct themes affecting faith and coping, including: dependence on belief and faith, providing purpose and meaning, establishing a connection with God by praying, establishing a connection with other individuals, and feeling a sense of gratitude and hope (7). Spiritual and religious practices may serve as a crucial means of defining and managing $\mathrm{PD}$ for these patients.

Spiritual distress is an integral component of palliative care assessments and treatments, necessitating the inclusion of spiritual counselors on palliative care teams. As PD is now an area for palliative care delivery, spiritual distress in both patients and caregivers, as well as the relationship between spirituality and palliative symptoms in PD, need to be better understood to optimize spiritual palliative treatment for PD patients. Goals of the present study are to (I) examine the correlation of patient spiritual distress with mood, caregiver engagement, disease status, and caregiver spirituality, and (II) determine whether spirituality modulates correlation of QOL and other predictors in PD. Results of the study can be used to inform the practice of spiritual counselors engaging with PD patients and caregivers, specifically in palliative care settings.

\section{Methods}

This cross-sectional study is based on the baseline data of a larger randomized, controlled trial, described in greater depth in a separate manuscript (8). This study was conducted through three medical institutions with currently operating outpatient palliative care clinics for $\mathrm{PD}$, and institutional ethics review board approval was received for each of the three study sites. Participants were recruited through specialized movement disorders programs, community neurologists, and self-referral from patients. One caregiver was enrolled for each participant, when available, by asking PD patients, "Could you please tell us the one person who helps you the most with your PD outside of clinic?".

\section{Participants}

Participants were eligible if they were fluent in English, over 40 years of age, and met criteria for diagnosis of $\mathrm{PD}$, progressive supranuclear palsy (PSP), corticobasal degeneration (CBD), multiple systems atrophy (MSA), or Lewy body dementia (LBD). Individuals also had to be assessed as high-risk for poor outcomes based on screening with the Palliative Care Needs Assessment Tool (PC-NAT) modified for PD. Participants were excluded based on the following factors: (I) immediate and urgent palliative care needs (these patients were not randomized into the larger trial and were offered appropriate services immediately); (II) unable or unwilling to commit to study procedures; (III) presence of additional chronic medical illnesses which may require palliative services (e.g., metastatic cancer); or (IV) already receiving palliative care and/or hospice services.

\section{Outcome measures}

All outcomes reflect baseline measurements only. The primary outcome measure for the study was the Functional Assessment of Chronic Illness Therapy - Spiritual Wellbeing (FACIT-Sp) $(9,10)$, a 12-item scale with responses scored on a 5-point Likert scale from 0 to 4 . The measure has three subscales: the sense of meaning, the feeling of peace, and the role of faith in illness. A summary score of spiritual well-being was calculated, ranging from 0 to 60 , with higher scores indicating greater spiritual well-being $(9,11)$.

\section{$Q O L$}

Because no QOL measure has been validated for a palliative PD population, several measures were used to assess QOL, including: the Parkinson's Disease Questionnaire 39 (PDQ39) (12), the McGill Quality of Life Questionnaire (McGill QOL) (13), the Patient-Reported Outcomes Measurement Information System (PROMIS-29) (14), and the Quality of Life in Alzheimer's Disease (QOL-AD) (15). 


\section{Mood and cognition}

The Hospital Anxiety and Depression Scale (HADS) measured self-reported anxiety and depression and has been validated in outpatient settings (16). Cognition was assessed with the Montreal Cognitive Assessment (MoCA), which is commonly used to assess cognitive status in $\mathrm{PD}$ populations (17).

\section{Advance care planning}

Participant possession of a completed Physician Order for Life Sustaining Treatment (POLST), Medical Orders for Scope of Treatment (MOST), or other advance directive (AD) was measured at a nominal ('yes' vs. 'no') level.

\section{Physical symptoms}

Physical symptoms, including current disease stage, were assessed by neurologists using the Unified Parkinson's Disease Rating Scale (UPDRS) (18). PD-specific palliative symptoms were measured by the Edmonton Symptom Assessment System Revised: Parkinson Disease (ESAS-rPD) (19).

\section{Statistical analyses}

Means and standard deviations were calculated for descriptive variables. Spearman or Kendall Tau correlations between total FACIT scores and continuous or ordinal demographic variables (age, disease duration, education categories, and income ranges) were determined. For non-ordinal categories, or when only a few categories existed, ANOVA type methods were considered. FACIT totals as well as Meaning, Peace, and Faith subscales were run in a Spearman correlation matrix with the following instruments: PDQ-39, HADS Anxiety, HADS Depression, PROMIS-29, McGill QOL, QOL AD, ESAS PD, MDS UPDRS 3, MoCA, Prolonged Grief PG 12, and the Palliative Performance Scale. Partial correlation was used to control for study site, age, sex, disease duration, presence of caregiver, involvement in support groups, education level, income, and cognitive status. Scale scores when items were missing were imputed using the half rule when applicable.

\section{Results}

\section{Patient characteristics}

Two-hundred and ten PD patients participated in the study, and over $83 \%(\mathrm{n}=175)$ had a caregiver. Mean patient age was 70.11 years $(\mathrm{SD}=8.15)$, and a majority of the sample $(\mathrm{n}=135,64 \%)$ was male. Approximately $91 \%(\mathrm{n}=159)$ of patients who had caregivers lived in the same household with them. Complete demographic data for the sample are detailed in Table 1 .

\section{Correlates of spiritual well-being}

Total FACIT score was available on 203 patients, and mean total FACIT score was 39.88 out of 60 (SD =9.64), with higher scores indicating greater spiritual well-being. With a total possible score of 20 for each subscale, patients reported average scores of $15.11(\mathrm{SD}=3.20, \mathrm{~N}=203)$ for Meaning, 12.87 (SD =3.42, N=203) for Peace, and 11.89 ( $\mathrm{SD}=4.88, \mathrm{~N}=200$ ) for Faith. Table 2 presents participant means and standard deviations for FACIT scores as well as the other outcome measures included in the study.

As presented in Table 3, spiritual well-being was positively correlated with older age, existence of a caregiver, involvement in support groups, and, to a lesser degree, income greater than $\$ 25,000$. Poorer spiritual health was associated with impairment in QOL (PDQ-39), increased anxiety (HADS Anxiety), increased depression (HADS Depression), increased non-motor symptoms (PROMIS-29), increased palliative symptoms (ESASPD), and prolonged grief (Prolonged Grief PG 12) (Table 4). Increased spiritual well-being was positively correlated with higher QOL as measured through the McGill QOL and Quality of Life AD scales. There was no association between patient FACIT scores and the MDS UPDRS or MoCA scores. There was no correlation between spiritual well-being and the ESASrPD in bivariate analyses; however, after controlling for potential confounders (age, sex, disease duration, involvement in support groups, education, income, and MoCA), higher levels of spirituality was associated with lower symptom burden as measured by the ESAS-rPD $(\mathrm{r}=-0.38, \mathrm{P}<0.0001)$.

Caregiver spirituality was positively correlated with patient spirituality, $r[168]=0.34, \mathrm{P}<0.0001$. Participants with a caregiver had significantly higher spiritual well-being $(106.90, \mathrm{SD}=316.00)$ than participants without caregivers (78.49, SD =316.00).

Multivariate analyses indicated consistent trends between variables and the FACIT subscales; however, for all analyses, the Faith subscale had weaker associations with mood and QOL variables than Peace and Meaning. 
Table 1 Participant characteristics

\begin{tabular}{|c|c|}
\hline Characteristic & $\begin{array}{c}\text { Number, mean } \pm \text { SD or } \\
\mathrm{n}(\%)\end{array}$ \\
\hline Age & $70.1 \pm 8.2$ \\
\hline Disease duration (months) & $115.4 \pm 81.3$ \\
\hline Caregiver age & $66.1 \pm 11.0$ \\
\hline Caregiving duration (months) & $68.5 \pm 62.8$ \\
\hline Sex (male) & $135(64.3)$ \\
\hline \multicolumn{2}{|l|}{ Race/ethnicity } \\
\hline African American & $3(1.4)$ \\
\hline Asian & $6(2.9)$ \\
\hline Native American & $1(0.5)$ \\
\hline White & $193(92.3)$ \\
\hline Mixed & $2(1.0)$ \\
\hline Other & $4(1.9)$ \\
\hline \multicolumn{2}{|l|}{ Education } \\
\hline Less than high school diploma & $13(6.3)$ \\
\hline High school diploma & $12(5.8)$ \\
\hline Some college & $30(14.4)$ \\
\hline Associate's degree & $15(7.2)$ \\
\hline Bachelor's degree & $49(23.6)$ \\
\hline More than a 4-year degree & $89(42.8)$ \\
\hline \multicolumn{2}{|l|}{ Marital status } \\
\hline Never married & $10(4.8)$ \\
\hline Married & $161(76.7)$ \\
\hline Separated & $4(1.9)$ \\
\hline Widowed & $14(6.7)$ \\
\hline Divorced & $19(9.0)$ \\
\hline Unknown & $2(1.0)$ \\
\hline \multicolumn{2}{|l|}{ Income } \\
\hline$\$ 0-19,999$ & $8(4.4)$ \\
\hline$\$ 20,000-29,999$ & $17(9.4)$ \\
\hline$\$ 30,000-39,999$ & $5(2.8)$ \\
\hline$\$ 40,000-49,999$ & $18(10.0)$ \\
\hline$\$ 50,000-59,999$ & $14(7.8)$ \\
\hline$\$ 60,000-74,999$ & $26(14.4)$ \\
\hline$\$ 75,000-99,999$ & $43(23.9)$ \\
\hline$\$ 100,000+$ & $48(26.7)$ \\
\hline Unknown & $1(0.6)$ \\
\hline Had deep brain stimulation surgery (yes) & $28(13.3)$ \\
\hline Involved in support groups (yes) & $110(53.1)$ \\
\hline
\end{tabular}

\section{Relationship between spiritual well-being and QOL}

Less patient spiritual wellbeing was associated with greater impairment in QOL, as measured by the PDQ-39 ( $\mathrm{r}=-0.41$, $\mathrm{P}<0.0001)$. This remained true for all FACIT subscales; however, the Faith subscale $(\mathrm{r}=-0.24, \mathrm{P}=0.002)$ had a weaker correlation than Peace $(\mathrm{r}=-0.48, \mathrm{P}<0.0001)$ and Meaning $(r=-0.41, \mathrm{P}<0.0001)$. For $\mathrm{QOL}$ as measured by the PROMIS-29, overall FACIT $(\mathrm{r}=-0.49, \mathrm{P}<0.0001)$ as well as subscale scores for Meaning $(r=-0.44, n=170, P<0.0001)$, Peace $(r=-0.54, \mathrm{P}<0.0001)$, and Faith $(\mathrm{r}=-0.29, \mathrm{P}=0.0002)$ mirrored associations with the PDQ-39. On both the McGill and QOL AD, higher scores indicate better QOL. Association trends between spiritual well-being and QOL remain consistent for these measures, with the strongest associations exhibited in the McGill QOL. The FACIT summary score was positively associated with QOL on the McGill $(r=0.63, P<0.0001)$, as were subscales Meaning $(\mathrm{r}=0.62, \mathrm{P}<0.0001)$, Peace $(\mathrm{r}=0.63, \mathrm{P}<0.0001)$, and Faith $(r=0.40, P<0.0001)$. Associations with the FACIT summary score $(r=0.46, \mathrm{P}<0.0001)$, Meaning $(\mathrm{r}=0.37, \mathrm{P}<0.0001)$, Peace $(r=0.55, \mathrm{P}<0.0001)$, and Faith $(\mathrm{r}=0.33, \mathrm{P}<0.0001)$ were slightly weaker with the QOL AD.

\section{Discussion}

Although previous research has qualitatively acknowledged the presence of a relationship between spirituality and Parkinson's disease management, this is the first known study to thoroughly detail the relationship between several domains of spirituality and the physical, mental, and palliative symptoms of $\mathrm{PD}$ as measured by standardized instruments. Although physical symptoms were not related to FACITSp scores, enhanced patient spiritual well-being was associated with less anxiety and depression. This contrasts with a previous study on spirituality and mood in PD using the Royal Free Interview (RFI) measure documenting no correlation between these variables (20). This discordance may reflect varied nuances between the measures, indicating that the FACIT may better capture spiritual and religious factors specifically associated with PD.

This study is unique in that it examines these relationships by subdomains of spirituality-Meaning, Peace, and Faith - in a group of persons with PD. Previous research examining these subdomains with cancer patients noted that Peace was only correlated with mental health (21); meaning correlated with both physical and mental health measures; and faith had a negative association with mental 
Table 2 Participant outcome measure characteristics

\begin{tabular}{lc}
\hline Measure & Number, mean \pm SD \\
\hline FACIT SP-12 & $39.9 \pm 9.6$ \\
FACIT SP Meaning & $15.1 \pm 3.2$ \\
FACIT SP Peace & $12.9 \pm 3.4$ \\
FACIT SP Faith & $11.9 \pm 4.9$ \\
PDQ-39 & $96.8 \pm 28.2$ \\
HADS Anxiety & $7.6 \pm 4.1$ \\
HADS Depression & $7.1 \pm 3.6$ \\
PROMIS-29 & $78.5 \pm 20.4$ \\
McGill QOL & $114.2 \pm 24.9$ \\
Quality of Life AD & $34.1 \pm 5.6$ \\
ESAS PD & $56.1 \pm 25.8$ \\
MDS UPDRS 3 & $40.3 \pm 18.7$ \\
MoCA & $23.8 \pm 4.9$ \\
Prolonged Grief PG 12 & $24.8 \pm 9.2$ \\
Palliative Performance Scale & $64.6 \pm 11.3$ \\
\hline
\end{tabular}

Table 3 FACIT SP-12 correlation with demographic variables

\begin{tabular}{lc}
\hline Characteristic & Correlation coefficient \\
\hline Age & $0.19^{\star \star}$ \\
Disease duration & -0.03 \\
Education & -0.10 \\
Income & 0.12 \\
\hline
\end{tabular}

**, $\mathrm{P}<0.01$.

health (10). Our results differ in that all three subscales had positive associations with mental health and actually no association with physical symptoms as measured through the MDS UPDRS. Although Faith had weaker associations with measures than the other subscales, it was still positively associated with better mental health and QOL, in contrast with a previous study in cancer patients. In the case of all three subscales, higher spirituality was associated with improved QOL despite marked motor impairment as measured by the MDS-UPDRS.

Robust research in oncology populations has long noted a relationship between spiritual well-being and healthrelated QOL. Delving further, one study found that spiritual well-being had a negative association with specific coping and adjustment styles, such as helpless/hopeless, anxious preoccupation, and avoidance, and it was positively associated with fatalism and a fighting spirit (22). A separate study in mixed long-term chronic illnesses in an Australian population noted the same positive trends between subscales and health-related QOL as the present study, and they also found that Meaning and Peace components were more strongly related to QOL than Faith (23). Their results indicated that employing a fatalistic coping style (e.g., accepting the diagnosis or stoic acceptance) in cancer treatment was positively correlated with Faith while being negatively associated with Meaning and Peace.

Some research indicates that religiosity in PD may be influenced through biological neurocognitive mechanisms affected by the disease (24); however, present findings indicate that the relationship between spirituality and PD is even more complex than previously understood. In addition to correlations with mood, increased spirituality was related to fewer non-motor symptoms and palliative symptoms, and reduced prolonged grief. Findings suggest we may be able to employ patient spirituality through meaning-making and peace to aid in reducing prolonged grief and to enhance patient coping with non-motor symptoms. These complex correlations with physical symptoms, mood, and cognitive factors allude to a multifaceted relationship between spirituality and health in PD, requiring additional research for a more comprehensive understanding of how spirituality can enhance patient care and coping in PD.

\section{Limitations}

The primary limitation of the present study is its crosssectional design, preventing the determination of causality in the clinical relationships discussed. Despite this, these data support a growing evidence base of the relationship between spiritual well-being and the health, mood, and QOL of PD patients, and they highlight the importance of including spiritual support on interdisciplinary and palliative care teams.

One of the original objectives of this study was to examine predictors of spiritual distress, which is defined by a FACIT-Sp score of $\leq 12$ (25). However, this aim could not be addressed, as the lowest FACIT-Sp score for patients was 16 , and the lowest for caregivers was 21 . Therefore, no spiritually distressed patients or caregivers were included in the sample or analyses. This narrow range of FACITSp scores may subsequently limit the generalizability of findings to patients experiencing significant spiritual distress, and additional research is needed to better 


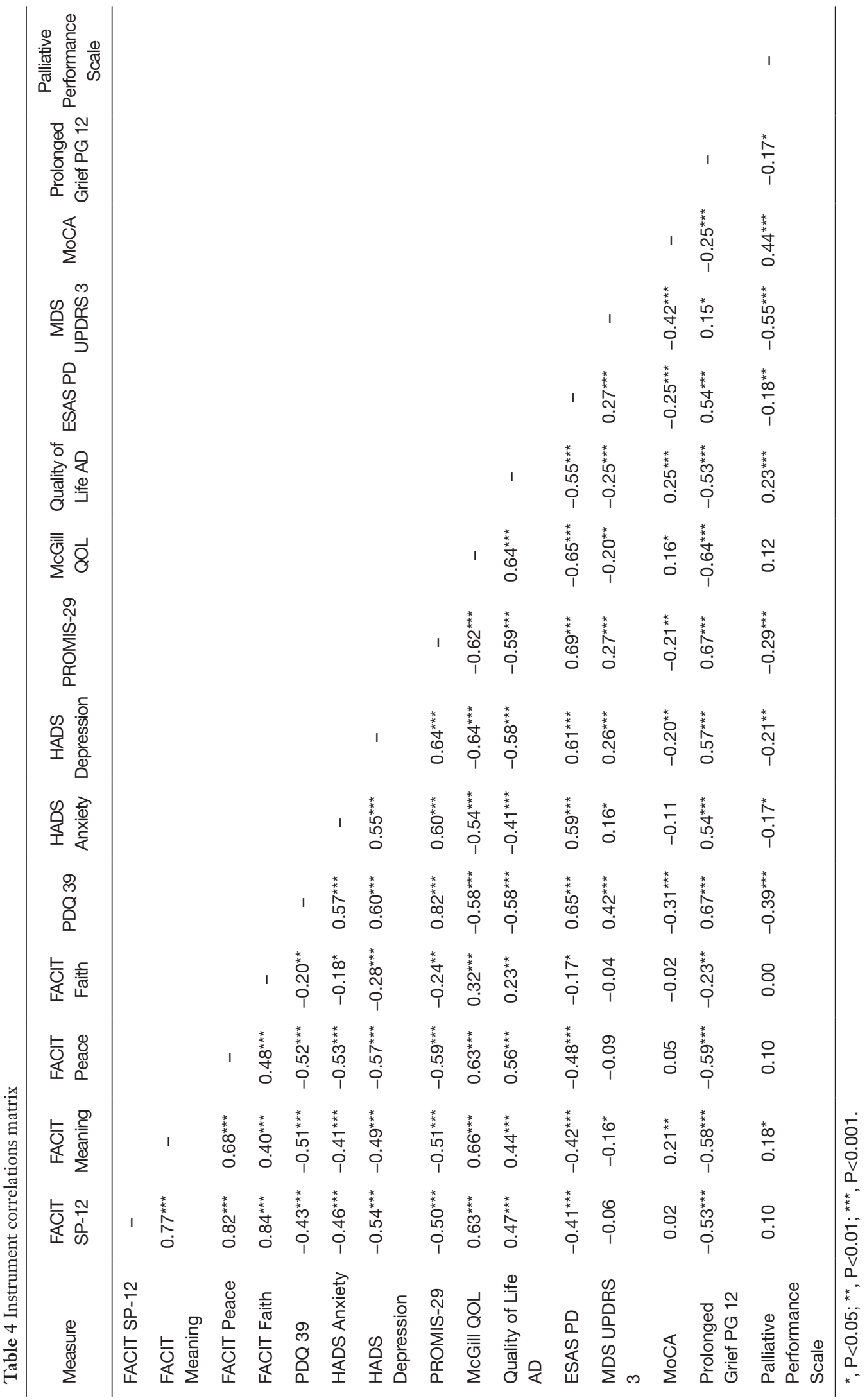


understand the predictors of poor spiritual wellbeing for palliative PD patients experiencing it.

\section{Conclusions}

The present study supports a growing body of literature emphasizing a relationship between spirituality and health, and understanding this association is particularly important for practitioners working with chronic illnesses, such as PD. Religion and spirituality may influence medical decision-making as well as patient coping strategies and attitudes (26). These results highlight the influences of spirituality in PD management, particularly the relationship between Meaning, Peace, and Faith and mood, palliative symptoms, and QOL.

A critical finding of this study is that spirituality may mitigate some of the physical and non-motor symptoms of PD (including anxiety and depression), and may result in enhanced QOL. As our field is researching pharmacologic means of treating these symptoms, spiritual assessments and supports may prove an empowering resource for patients in their treatment that is consistent with patient values and with little risk. Based on this evidence, clinicians treating individuals with PD should consider spiritual assessments in their practice and should provide patients with access to spiritual counselors experienced in helping those with chronic medical conditions when possible. These data further support the holistic, interdisciplinary care provided to PD patients through palliative care teams offering chaplaincy support to patients.

\section{Acknowledgments}

Funding: Research reported in this publication was funded through a Patient-Centered Outcomes Research Institute (PCORI) Award (IHS-1408-20134). All statements in the publication are solely the responsibility of the authors and do not necessarily represent the views of the PCORI, its Board of Governors or Methodology Committee.

\section{Footnote}

Conflicts of Interest: The authors have no conflicts of interest to declare.

Ethical Statement: The authors are accountable for all aspects of the work in ensuring that questions related to the accuracy or integrity of any part of the work are appropriately investigated and resolved. Institutional ethics review board approval was received for each of the three study sites (IRB 15-0814). Written informed consent was obtained from the patient for publication of this manuscript and any accompanying images.

\section{References}

1. Hermanns M, Deal B, Haas B. Biopsychosocial and Spiritual Aspects of Parkinson Disease: An Integrative Review. J Neurosci Nurs 2012;44:194-205.

2. Rafferty KA, Billig AK, Mosack KE. Spirituality, Religion, and Health: The Role of COmmunication, Appraisals, and Coping for Individuals Living with Chronic Illness. J Relig Health 2015;54:1870-85.

3. Rowe MM, Allen RG. Spirituality As a Means of Coping with Chronic Illness. Am J Health Stud 2004;19:62.

4. Hills J, Paice JA, Cameron JR, et al. Spirituality and Distress in Palliative Care Consultation. J Palliat Med 2005;8:782-8.

5. Finseth TA, Hedeman JL, Brown RP, et al. Self-Reported Efficacy of Cannabis and Other Complementary medicine Modalities by Parkinson's Diseae Patients in Colorado. Evid Based Complement Alternat Med 2015;2015:874849.

6. Reynolds D. Spirituality as a Coping Mechanism for Individuals With Parkinson's Disease. J Christ Nurs 2017;34:190-4.

7. Bingham V, Habermann B. The Influence of Spirituality on Family Management of Parkinson's Disease. J Neurosci Nurs 2006;38:422-7.

8. Kluger BM, Katz M, Galifianakis N, et al. Does Outpatient Palliative Care Improve Patient-Centered Outcomes in Parkinson's Disease: Rationale, Design, and Implementation of a Pragmatic Comparative Effectiveness Trial. Contemp Clin Trials 2019;79:28-36.

9. Canada AL, Murphy PE, Fitchett G, et al. A 3-Factor Model for the FACIT-Sp. Psychooncology 2008;17:908-16.

10. Murphy PE, Canada AL, Fitchett G, et al. An Examination of the 3-Factor Model and Structural Invariance Across Racial/Ethnic Groups for the FACITSp: A Report from the American Cancer Society's Study of Cancer Survivors - 11 (SCS-II). Psychooncology 2010;19:264-72.

11. Peterman AH, Fitchett G, Brady MJ, et al. Measuring Spiritual Well-Being in People With Cancer: The Functional Assessment of Chronic Illness Therapy Spiritual Well-Being Scale (FACIT-Sp). Ann Behav Med 2002;24:49-58. 
12. Jenkinson C, Fitzpatrick R, Peto V, et al. The Parkinson's Disease Questionnaire (PDQ-39): development and validation of a Parkinson's disease summary index score. Age ageing 1997;26:353-7.

13. Cohen SR, Mount BM, Strobel MG, et al. The McGill Quality of Life Questionnaire: A Measure of Quality of Life Appropriate for People with Advanced Disease. A Preliminary Study of Validity and Acceptability. Palliat Med 1995;9:207-19.

14. Cella D, Riley W, Stone A, et al. Initial Item Banks and First Wave Testing of the Patient-Reported Outcomes Measurement Information System (PROMIS) Network: 2005-2008. J Clin Epidemiol 2010;63:1179-94.

15. Thorgrimsen L, Selwood A, Spector A, et al. Whose Quality of Life Is It Anyway?: The Validity and Reliability of the Quality of Life-Alzheimer's Disease (QoL-AD) Scale. Alzheimer Dis Assoc Disord 2003;17:201-8.

16. Zigmond AS, Snaith RP. The Hospital Anxiety and Depression Scale. Acta Psychiatrica Scandinavica 1983;67:361-70.

17. Dalrymple-Alford JC, MacAskill MR, Nakas CT, et al. The MoCA: Well-Suited Screen for Cognitive Impairment in Parkinson Disease. Neurology 2010;75:1717-25.

18. Goetz CG, Tilley BC, Shaftman SF, et al. Movement Disorder Society-Sponsored Revision of the Unified Parkinson's Disease Rating Scale (MDS-UPDRS): Scale Presentation and Clinimetric Testing Results. Mov Disord 2008;23:2129-70.

19. Miyasaki J, Long J, Mancini D, et al. Palliative Care for

Cite this article as: Prizer LP, Kluger BM, Sillau S, Katz M, Galifianakis N, Miyasaki JM. Correlates of spiritual wellbeing in persons living with Parkinson disease. Ann Palliat Med 2020;9(Suppl 1):S16-S23. doi: 10.21037/apm.2019.09.13
Advanced Parkinson Disease: An Interdisciplinary Clinic and New Scale, the ESAS-PD. Parkinsonism Relat Disord 2012;Suppl 3:S6-9.

20. Giaquinto S, Bruti L, Dall'Armi V, et al. Religious and Spiritual Beliefs in Outpatients Suffering from Parkinson Disease. Int J Geriatr Psychiatry 2011;26:916-22.

21. Schultz M, Meged-Book T, Mashiach T, et al. Distinguishing Between Spiritual Distress, General Distress, Spiritual Well-Being, and Spiritual Pain Among Cancer Patients During Oncology Treatment. J Pain Symptom Manage 2017;54:66-73.

22. Cotton SP, Levine EG, Fitzpatrick CM, et al. Exploring the Relationships Among Spiritual Well-Being, Quality of Life, and Psychological Adjustment in Women with Breast Cancer. Psychooncology 1999;8:429-38.

23. Whitford HS, Olver IN, Peterson MJ. Spirituality as a Core Domain in the Assessment of Quality of Life in Oncology. Psychooncology 2008;17:1121-8.

24. McNamara P, Durso R, Brown A, et al. The chemistry of religiosity: Evidence from Patients with Parkinson's Disease. In: McNamara P. editor. Where God and Science Meet: How Brain and Evolutionary Studies Alter Our Understanding of Religion. 2006;2:1-14.

25. McClain CS, Rosenfeld B, Breitbard W. Effect of Spiritual Well-Being on End-of-Life Despair in Terminally-Ill Cancer Patients. Lancet 2003;361:1603-7.

26. Koenig HG. Religion, Spirituality, and Medicine: Research Findings and Implications for Clinical Practice. South Med J 2004;97:1194-200. 